\title{
A DIGITAL SELF EXCITED LOOP FOR ACCELERATING CAVITY FIELD CONTROL*
}

\author{
T. Allison, J. Delayen, C. Hovater $\$$, J. Musson, and T. Plawski, TJNAF, Newport News, VA, USA
}

\begin{abstract}
We have developed a digital process that emulates an analog oscillator and ultimately a self excited loop (SEL) for field control. The SEL, in its analog form, has been used for many years for accelerating cavity field control. In essence the SEL uses the cavity as a resonant circuit -much like a resonant (tank) circuit is used to build an oscillator. An oscillating resonant circuit can be forced to oscillate at different, but close, frequencies to resonance by applying a phase shift in the feedback path. This allows the circuit to be phased-locked to a master reference, which is crucial for multiple cavity accelerators. For phase and amplitude control the SEL must be forced to the master reference frequency, and feedback provided for in both dimensions. The novelty of this design is in the way digital signal processing (DSP) is structured to emulate an analog system. While the digital signal processing elements are not new, to our knowledge this is the first time that the digital SEL concept has been designed and demonstrated. This paper reports on the progress of the design and implementation of the digital SEL for field control of superconducting accelerating cavities.
\end{abstract}

\section{INTRODUCTION}

Presently the CEBAF Accelerator at Jefferson Lab is a $6 \mathrm{GeV}$ five pass electron accelerator consisting of two superconducting linacs joined by independent magnetic transport arcs. In the future it is intended to increase the energy to $12 \mathrm{GeV}$. This is to be accomplished by adding five new $98 \mathrm{MeV}$ cryomodules to each linac (10 total). To control the RF for these new cryomodules a new Low Level RF (LLRF) system is being designed. Cavity field control is maintained digitally using an Altera FPGA which contains the feedback algorithm. The system incorporates digital down conversion, using quadrature under-sampling at an IF frequency of $70 \mathrm{MHz}$. A VME based system is being used as a prototype but it is planned to migrate to a stand alone EPICS IOC using a PC104 module.

Three upgrade cryomodules have been produced consisting of a new 7 cell cavity design and incorporating a new PZT mechanism [1]. Two of these modules have been tested and installed, one in the CEBAF accelerator and the other in the Jefferson Lab FEL. Initial LLRF testing was done last summer [2]. In these tests digital LLRF control was demonstrated to meet field control requirements up to $16.7 \mathrm{MV} / \mathrm{m}$. For these tests a simple Generator Driven Resonator (GDR) algorithm was used. In this paper we report on tests using a Digital Self Excited Loop (DSEL) Algorithm.

* Authored by Jefferson Science Associates, LLC under U.S. DOE Contract No. DE-AC05-06OR23177. The U.S. Government retains a non-exclusive, paid-up, irrevocable, world-wide license to publish or reproduce this manuscript for U.S. Government purposes.

\$ hovater@jlab.org

\section{Self Excited Loop}

An SEL algorithm has one distinct advantage over a GDR it immediately excites a cavity regardless of frequency. For a superconducting cavity (sc) that has a large Lorentz detuning coefficient this allows an RF system to recover a cavity to the operational gradient with out having to tune it. In a CW RF system this is optimum. Even if the cavity has been detuned many bandwidths, the frequency difference between the reference and the cavity can be obtained and the cavity quickly tuned back. This is accomplished without the use of hunting algorithms or employing a Numerically Controlled Oscillator (NCO) which GDR systems must use.

The self excited loop was first presented by Delayen [3] and has been used since for control of superconducting accelerator structures. The heavy ion accelerators at Argonne, JAEA Tokai, Canberra and the electron accelerator at Darmstadt both use an analog version of the SEL. It is principally used for structures operated cw, with external Q's above $1 \times 10^{7}$, but can be used for any resonant structure. Figure 1 shows a block diagram of an SEL. In its simplest form it is an oscillator (amplifier driving a resonant circuit) with positive feedback. In this form it will naturally track the cavity frequency given that the amplifiers bandwidth is sufficiently larger than the cavity bandwidth. Amplitude control is obtained by limiting the feedback amplitude and then providing an external set point which can be compared to the cavity signal for additional amplitude control. The circuit can be phase-locked to a reference by comparing the cavity signal to the reference and feeding back into the SEL. The phase angle forces the loop frequency and phase to lock to the reference. This is best done adding a signal in quadrature instead of simply introducing a phase shift. The cavity frequency must be tuned close enough to the reference such that the amplifier does not saturate. Optimally for RF power reasons this is less than half a cavity bandwidth.

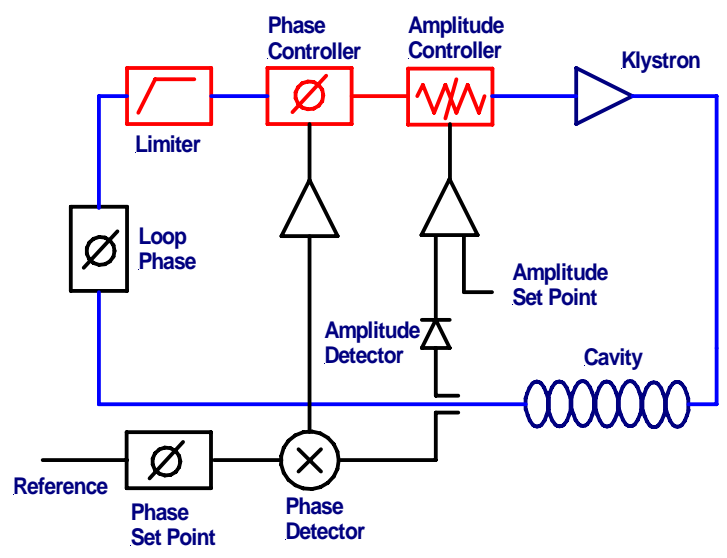

Figure 1: SEL Block Diagram 


\section{CONVERSION TO DIGITAL DOMAIN}

The idea of building a digital SEL has been around for some years. In one case this has partially been done. At TRIUMF a hybrid analog-digital SEL that utilizes a baseband DSP PID loop was developed [4]. The system also incorporates a PLL-VCO and the system can switch back and forth between the two modes. Our system takes it to the final step incorporating direct digital conversion at an IF frequency of $70 \mathrm{MHz}$. All processing would then occur at a multiple of the clock frequency, $56 \mathrm{MHz}$. The red components in figure 1 are the parts that must be digitized. The SEL algorithm was developed on a modified normal conducting LLRF controller [2]. It is a VME based digital controller consisting of an Altera FPGA. The algorithm was bench tested using a cavity simulator (crystal filter) that by frequency conversion mimicked a cavity with a $Q_{\text {ext }}$ of $10^{6}$.

Initial efforts began as an extension of the GDR based system. The first task was to demonstrate a digital oscillator that would track a cavity. Given the discrete nature of a digital system there was some concern that the noise buildup needed for an oscillator to start would be hindered by the ADC resolution. In addition there was a strong bias to oscillate at the $70 \mathrm{MHz}$ harmonic of the clock frequency not the cavity frequency. To do this the GDR system was programmed to pass the signal straight through. Loop phase was then adjusted with an external loop phase shifter. Results were encouraging and proved that a digital SEL was possible. Operating around a spare $499 \mathrm{MHz}$ deflecting cavity we observed oscillation and upon detuning the cavity the frequency tracked.

The challenge became coding a limiter and adding elements of phase and amplitude control i.e. the red components of figure 1. Given that the GDR based system was in the I\&Q domain we started down this path. A SystemVue model was developed to guide the DSP effort and help the FPGA logic design. The limiter effectively was a digital automatic gain controller. In addition a microphonic compensator was added after the limiter to correct for cavity detuning [3]. The results were mixed, but proved that the concept would work (small control bandwidth and dynamic range). Many of the issues arose with scaling and the PI constants of the AGC.

At this point we moved some of the digital signal processing out of the I/Q domain into phase and amplitude. Use of a CORDIC made this transformation rather easy. The CORDIC is an iterative algorithm that converts the signal from Cartesian (IQ) to Polar (phase and amplitude). For signal processing the phase and amplitude domain has the advantage of simpler logic operations (very important for an FPGA). The CORDIC also allowed the firmware to handle a spinning vector which an SEL must do when not locked. In addition by separating the magnitude and phase the limiter function can be easily realized. Figure 2 shows a block diagram of the control logic. After digitization (I\&Q) the cavity field signal is fed through FIR filters before the CORDIC. Once in this domain various operational modes can be obtained.

1. Tone: The simplest case is the straight forward tone. The Amplitude and Phase loop are open and amplitude control is through a set point. A tone is then generated at $1497 \mathrm{MHz}$ frequency which is determined by the reference clock.

2. SEL: To switch to SEL mode the phase loop from the cavity is re-connected (feedback) and a loop phase shifter added. In this mode the loop resembles a PLL-VCO in that it tracks the cavity frequency. Amplitude control is the same as the tone-mode. Controlling the amplitude in this fashion has the effect of a perfect limiter.

3. Microphonics Compensation: Figure 3 shows the method for compensating for microphonics using an SEL. By obtaining a phase error signal and applying it proportionally to the compensation algorithm in the IQ domain the effect of microphonics on cavity field can be minimized. Gradient is adjusted similarly to the tone. For this to work the loop must be on resonance.

4. Amplitude Control: In this case a PID controller is added in the amplitude feedback path. This can be operated in SEL or compensation mode.

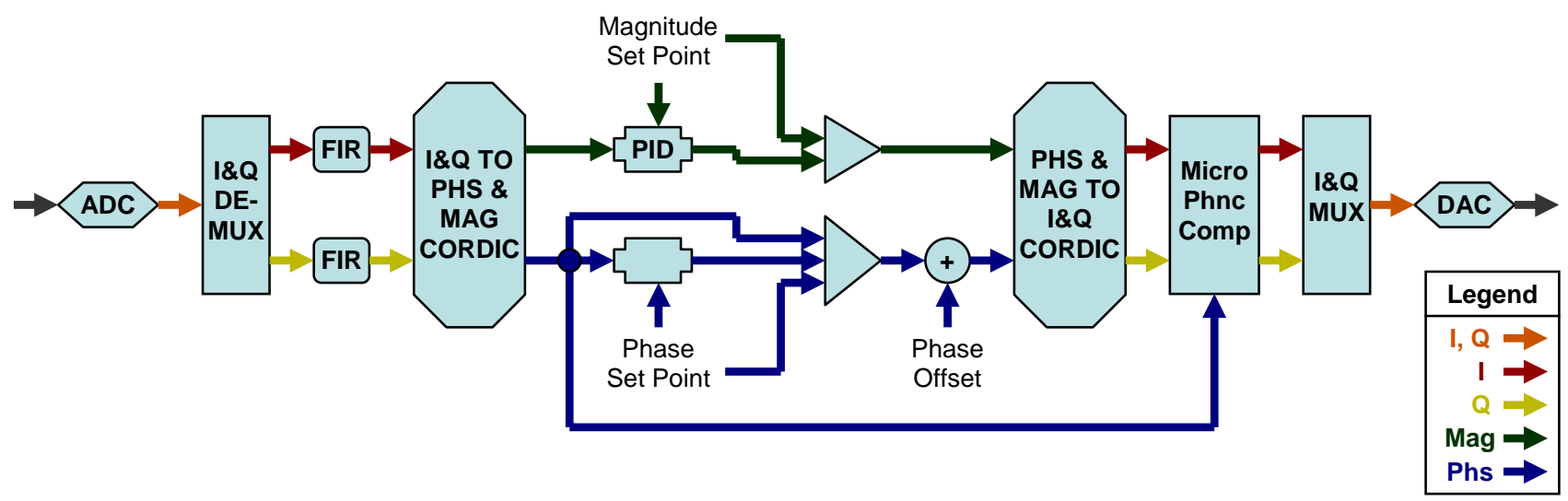

Figure 2 Algorithm Block Diagram 
After signal processing another CORDIC is used to bring the signals back into the IQ domain. The signals are then passed through the microphonics compensator (on or off) and then through a MUX. Finally a single DAC processes the signal and generates the IF needed for up conversion to the cavity frequency.

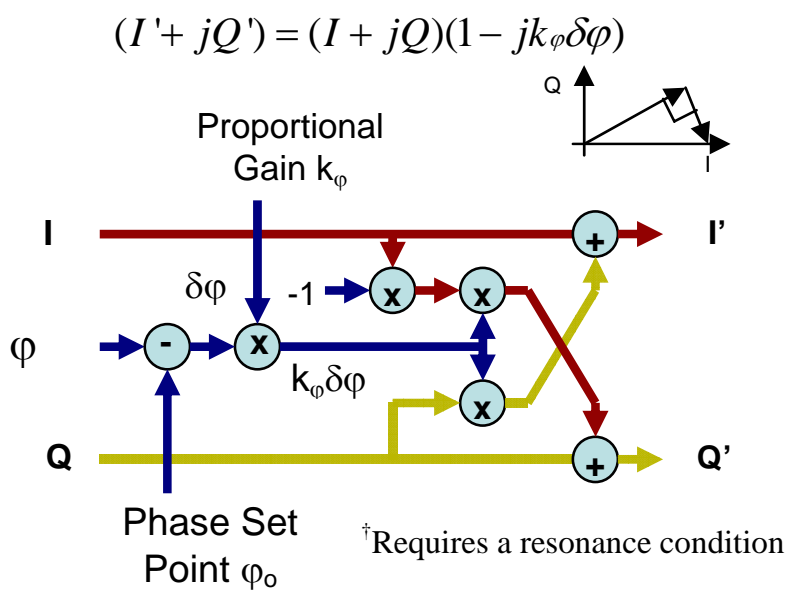

Figure 3 Microphonic compensator to provide phase-lock.

\section{CAVITY TESTS AND MEASUREMENTS}

SC cavity tests were made on an upgrade cryomodule using the Jefferson Lab Free Electron Laser (FEL). We began measurements first at low power $(\sim \mathrm{mV} / \mathrm{m})$ and then at operational levels $(\sim \mathrm{MV} / \mathrm{m})$. For high power tests the existing LLRF system acted as the cavity interlocks. The drive and cavity signals were then routed to the digital system. Additionally a separate LO and clock were used making testing simpler.

In SEL mode the system quickly found the cavity resonance. This was tested a number of ways. First the LO source was detuned $+/-50 \mathrm{kHz}$ while observing the cavity signal using a spectrum analyzer. The spectrum analyzer frequency never changed. Next the cavity was mechanical detuned by $1.3 \mathrm{kHz}$ while observing the frequency. This time the frequency changed as the resonance frequency changed. Finally the LLRF system was turned off and the LO source detuned $10 \mathrm{kHz}$ and turned back on. Again the system immediately found resonance.

In an SEL it is possible to achieve phase and gradient control using a microphonic compensator scheme that uses the phase error signal and applies a proportional amount to the I and Q vectors (Fig 3). Turning on the compensator and adjusting the proportional gain allowed us to reduce the phase error from $0.75^{\circ} \mathrm{rms}$. to $0.11^{\circ} \mathrm{rms}$ error (our specification is $0.5^{\circ} \mathrm{rms}$.). This was measured using an Agilent Signal Source Analyzer (Fig. 4). The error was dominated at the low frequency between 1-10 $\mathrm{Hz}$ by the Local Oscillator phase noise and is excluded in the measurement. In the CEBAF accelerator the master oscillator LO has lower phase noise and this should not be an issue. In SEL mode the cavity amplitude error was $0.1 \%$ which was 3 times our target specification of
0.045\% rms.. This we believe is caused by system nonlinearities (amplifiers, mixers etc.). To achieve the required amplitude field control we applied a magnitude PID loop to the compensated SEL. By applying proportional control we were able to achieve our amplitude field control specification of $0.045 \%$.

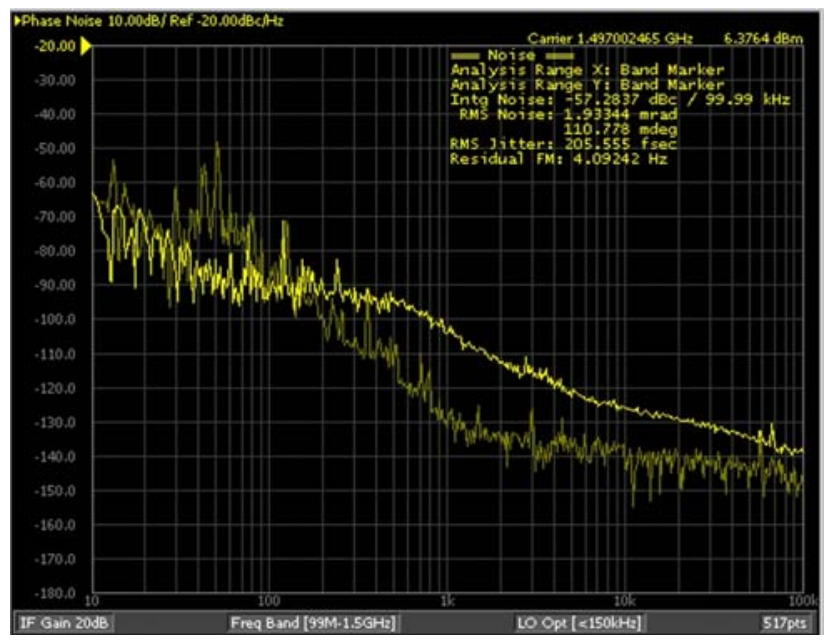

Figure 4 Phase noise with compensator on

\section{SUMMARY}

The ability to incorporate an SEL algorithm into a digital LLRF system has been demonstrated. The utility of this feature has not yet been fully realized. When operational, cavity recovery from faults will be tuner-less. While this has not been an issue at low gradients, $\sim 5$ $\mathrm{MV} / \mathrm{m}$, at high gradients, $20 \mathrm{MV} / \mathrm{m}$, like those needed for the upgrade cryomodules the Lorentz detuning will be many bandwidths, making cavity turn-on problematic with out some tuner based compensation or other algorithmic solution. The SEL solves this problem and allows cavity recovery from cryogenic trips (cavities have been known to detune 1000's of $\mathrm{Hz}$ ). Other applications such has He processing can also be implemented in situ with out additional electronics.

The SEL algorithm is still being refined. Future tests are planned with enhanced operability i.e. a resonance control algorithm to keep the cavity within $1 \mathrm{~Hz}$ of the reference. Stay tuned.

\section{REFERENCES}

[1] E. Daly, et al, "Improved Prototype Cryomodule for the CEBAF $12 \mathrm{GeV}$ Upgrade”, PAC 2003 Proceedings, Portland OR, USA

[2] C. Hovater et al, "High Gradient Operation with the CEBAF Upgrade RF Control System” LINAC 2006 Proceedings, Knoxville TN, USA

[3] J. R. Delayen, "Phase And Amplitude Stabilization of Superconducting Resonators”, PhD Thesis, California Institute of Technology, 1978.

[4] K. Fong et al, RF Control System For ISAC II Superconducting Cavities, PAC 2003 Proceedings. 